\title{
Diagnostic and Therapeutical Tinnitus System Based on Fuzzy Rough Set
}

\author{
Ma Xian-Min \\ College of Electrical and Control Engineering \\ Xi' an University of Science \&Technology \\ Xi'an, 710054, China \\ e-mail: maxm@xust.edu.cn
}

\begin{abstract}
A novel distributed system for the diagnostic and therapeutical tinnitus is introduced based on the fuzzy rough set theory in this paper. The cause of the tinnitus diseases is analyzed, and the prime symptoms of disorders of the inner ear are classified. According to a ringing or booming sensation in one or both ears, the problem of the processing model is built based on fuzzy inference, and the remedial sound intensity level of the curative tinnitus is discussed. The particular requirements and characteristics of the cure system are analyzed. Research results reveal that, based on the fuzzy rough set theory, the fuzzy membership functions can be explicitly interpreted and the extracting diagnosis rules from fault data are feasible.
\end{abstract}

Keywords- diagnosis; therapeutical tinnitus; fuzzy inference; rough set

\section{INTRODUCTION}

Tinnitus denotes a ringing or booming sensation in one or both ears and is a symptom of an ear infection or Meniere's disease, which is one of the most common diseases in people. There are many methods to cure the diseases. But the reason of the tinnitus is not still clear; these conventional therapeutical methods have some difficulty to look for the correct remedial approach for the patients because the tinnitus sign is multifarious. Much symptom from the patients is uncertain. The knowledge acquisition is the technique bottleneck for the traditional Chinese Medicine ${ }^{[1]}$. According to the experiential opinion of the ear experts, there are many tinnitus data that can be mined as the rules for judge the type of the era disease. So in this paper, fuzzy logical set is used to inference for the tinnitus, and the rough set theory is adopted to reduce the rule clusters, because the rough set is an emerging tool to extract knowledge from a vast volume data. Knowledge acquisition technique based on rough set is just suitable for these discrete data ${ }^{[2]}$. In this paper, the principle of the novel distributed system based on the fuzzy rough set theory for the diagnostic and therapeutical tinnitus is introduced, and the diagnostic and curing process is described. The cause of the tinnitus diseases is analyzed, and the prime symptoms of disorders of the inner ear are classified. According to a ringing or booming sensation in one or both ears, the problem of the processing model is built based on fuzzy inference, and the remedial sound intensity level of the curative tinnitus is discussed. The particular requirements and characteristics of the cure system are analyzed.

\section{Diagnosis Tinnitus System}

According to the technique requirements, the novel distributed system based on the fuzzy rough set theory for the diagnostic and therapeutical tinnitus is composed of the master computer and eight transducers. Therefore, one master computer can supervise the eight transducers through the field bus. Ear states are detected by the sensor so that the current condition of ears is monitored by the master computer in this system. The real time distributed system of the diagnostic and therapeutical tinnitus is shown in Fig.1.

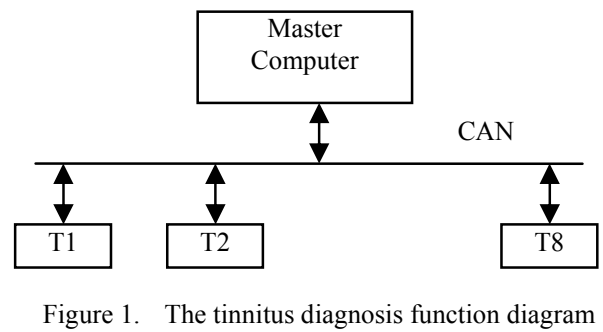

In Fig.1, $\mathrm{T}$ denotes the transducer which is composed of the sensor and remedial instruments. There are eight transducers i.e. $\mathrm{T} 1, \mathrm{~T} 2, \mathrm{~T} 3, \mathrm{~T} 4, \mathrm{~T} 5, \mathrm{~T} 6, \mathrm{~T} 7$, and $\mathrm{T} 8$ in the distributed real time diagnostic and therapeutical tinnitus system. The master computer has four functions such as investigation, recognition, healing and information. The doctor asks the many questions to the patients. From the reply and answer of the patients, the doctor judges the tinnitus type with the help of the transducer and detecting instruments and selects the suitable scheme to curing the ear disease. All the information of the patients is saved as the history records in the database of the master computer. Therefore the diagnostic and therapeutical tinnitus system has many advantages such as supervising, diacrisis, treatment and consultation. So the diagnostic and therapeutical tinnitus system can carry out the pure tone threshold test, tonality matching, sensitivity adjustment, tinnitus sound hodgepodge experiment, minimum audio masking curve clinic test and so on. Many other test functions can also be finished in the distributed system.

The tinnitus reasons are very complex, so the symptoms are also intricate. According expert experience, the fuzzy logical theory is adopted to judge which kind of the era disease. And the rule sets are reduced based on the rough set theory. 


\section{BASIC ROUGH SET}

Rough set theory uses the information table to express and process knowledge. Therefore data sets are usually given in the table form, and this data table is called an information system. In rough set theory, the knowledge is considered as the ability to classify the objects and described with the attribute and property value ${ }^{[3]}$. A knowledge expression system can be expressed as a fourtuple $S=(U, R, V, f)$, where $U=\left\{x_{1}, x_{2}, \ldots, x_{n}\right\}$ is a set of finite and nonempty objects, called the universe, $R$ is a set of the object attributes, $V$ is a set of the attribute values, $f$ is an information function $f: U \times R \rightarrow V$. If the attribute set is divided into condition attribute $C$ and decision attribute $D$, the information system is also called a decision table.

For given $x, X \subseteq U, R$ is an equivalence relation on $U$. When $X$ can be precisely described by the attribute of $R, X$ is called $R$ discernibility. Otherwise, $X$ is $R$ indiscernibility. $R$ definable set is a subset of the domain, and it can be precisely defined in the knowledge base $K=(U, R)$, so it is also called as $R$ accuracy set. But $R$ indiscernibility set can not be defined in the knowledge base, and is known as $R$ inaccuracy set or rough set.

With arbitrary attributes $K \subseteq R$, there is an associated indiscernibility relation $I N D(K)$. For given an equivalence relations $\mathrm{R} \in I N D(\mathrm{~K})$ if $X$ is a $R$ accuracy set, then the set $\mathrm{X} \subseteq \mathrm{U}$ is called as the accuracy set in $K$. For any $R \in \mathrm{IND}$ $(K)$, if $X$ always is $\mathrm{R}$ rough sets, then $X$ is called as rough set in $K$. An indefinable relation is an equivalent relation that satisfies reflexivity, symmetry and transitivity. Equivalence classes generated by $K$ are also called $K$ elemental granules, or $K$ information granules. The set of elemental granules forms a concept system, which is used to characterize arbitrary subsets in the information system. The set of $U \mid R$ express a group of mutual exclusive basic equivalence classes that $U$ is divided with $R$, and is called as basic set. The rough set can be approximately described through two accuracy sets, namely, the upper approximation $R^{-}(X)$ and lower approximation $R_{-}(X)$. Given knowledge base $K=(U, R)$, for each subset $X \subseteq U$ in the information system, and an equivalence relation $R \in I N D(K)$, two unions of elemental granules are associated with:

$$
\begin{gathered}
R_{-}(X)=\bigcup\{Y \in U / R \mid Y \subseteq X\} \\
R^{-}(X)=\bigcup\{Y \in U / R \mid Y \cap X \neq \varnothing\}
\end{gathered}
$$

The $R_{-}(X)$ is also called a positive region $\operatorname{POS}_{R}(X)$. If $R_{-}$ $(X)=R^{-}(X)$, the concept $X$ is a definable set, which means the concept $X$ can be perfectly characterized with the knowledge $K$. Otherwise, $X$ is indiscernibility. An indefinable set is known as a rough set. Similarly $N E G_{R}(X)$ is denoted as $R$ negative domain of $X$, and $N E G_{R}(X)=U-R^{-}(X)$ or $R^{-}(X)=P O S_{R}(X) \cup B N D_{R}(X)$, where $B N D_{R}(X)=R^{-}(X)-R_{-}(X)$ is defined as $R$ bounded domain of the approximations. Judged by knowledge $R$, in $R^{-}(X)$ or $\operatorname{POS}_{R}(X)$ sets there are elements of $U$, which certainly belong to $X$ sets, and $R(X)$ are sets whose elements probably be owned by $X$. In $B N D_{R}(X)$ sets the elements are neither $X$ nor $\bar{X}$, and $N E G_{R}(X)$ are sets whose elements certainly do not belong to $X$. As a definable set, the boundary is empty, as shown in Fig.2.

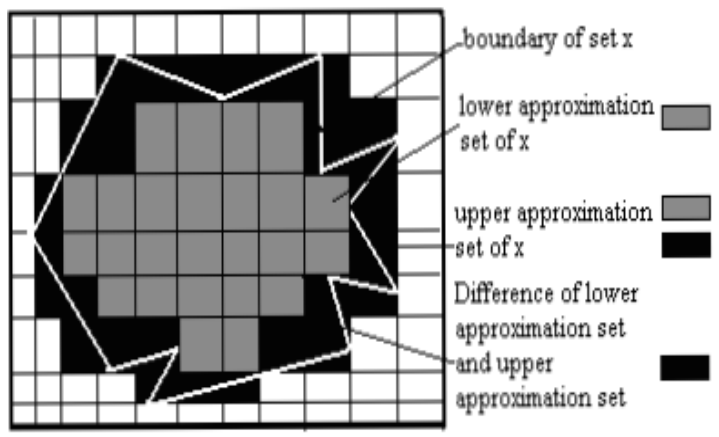

Figure 2. Rough set approximation

The inaccuracy of rough set is caused from existence of boundary domain. The greater of the boundary domain of sets, the lower its accuracy is. The approximate accuracy of sets $X$ defined by equivalence relation $R$ is as:

$$
a R(X)=|R-(X)| /|R-(X)|
$$

In this expression, $X \neq \varnothing,|X|$ expresses the radix of sets $X$. According to the accuracy definition, the $R$-roughness of $X$ is defined as:

$$
\rho R(X)=1-a R(X)
$$

Reduction theory is the crux of rough set, and the redundant or unnecessary attribute can be cancelled for simplified knowledge. A relative reduction is an independent subset of condition attributes. For a given decision table, there is usually more than one relative reduction, and the intersection of all the relative reduction is called the relative core. If there is a attribute $W \in R$, the indispensable attribute is $I N D(R) \neq I N D(R-\{W\})$. All indispensable relationship sets are called core of $R$ :

$$
\operatorname{CORE}(R)=\cap R E D(R)
$$

where, $R E D(R)$ is the all simplified sets.

\section{EXTRACTION RULES WITH FUZZY SET THEORY}

Tinnitus is often results from the some obstacle in the sound transmission channel of the ears. The tinnitus can be classified as two types, i.e. subjectivity and objectivity ear diseases. The subjectivity tinnitus is focused in the paper. After professor L.A.Zadeh proposed fuzzy set theory in 1965, fuzzy set theory has been widely used in many domains such as artificial intelligence, pattern recognition etc for the processing uncertainty and imprecision [4]. According to the Fuzzy set theory, the attribute-value table is adopted in the subjectivity tinnitus information system. In the attribute-value table the rows denote the objects in the domain of discourse, and the columns represent the attributes. In order to realize the reasonable inference, the ear pathema has many symptoms shown in Table 1 . 
TABLE I. TINNITUS SYMPTOM

\begin{tabular}{|l|l|}
\hline \multicolumn{1}{|c|}{ Symptom } & \multicolumn{1}{c|}{ Illness } \\
\hline Jam feeling & Tympanitis \\
\hline Dull in sound & Foreign matter \\
\hline Vertigo & Meniere's disease \\
\hline Some medicine & Medicinal syrigmus \\
\hline Noisy working & Employment syrigmus \\
\hline
\end{tabular}

According to the tinnitus serious degree, the sound press levels are classified as shown in Table 2.

TABLE II. SOUND PRESS LEVELS

\begin{tabular}{|c|c|}
\hline Sign & Level \\
\hline common & ZE \\
\hline mild & PS \\
\hline moderate & PM \\
\hline severe & PB \\
\hline terrible & PV \\
\hline
\end{tabular}

Regarding the membership function of tinnitus symptoms, there are five levels, namely, PV, PB, PM, PS, $Z E$. The set of linguistic term is $\{P V, P B, P M, P S, Z E\}$. The variable universe of sound loudness grate discourse is defined as $\{8,7,6,5,4,3,2,1,0\}$ as shown in Table 3 .

TABLE III. SOUND LOUDNESS GRATE

\begin{tabular}{|c|c|}
\hline Indication & Grate \\
\hline no tinnitus & 0 \\
\hline ordinary & 1 \\
\hline a little & 2 \\
\hline mild & 3 \\
\hline moderate & 4 \\
\hline severe & 5 \\
\hline terrible & 6 \\
\hline very terrible & 7 \\
\hline deaf & 8 \\
\hline
\end{tabular}

The membership function of the tinnitus symptoms is shown in Fig. 3.

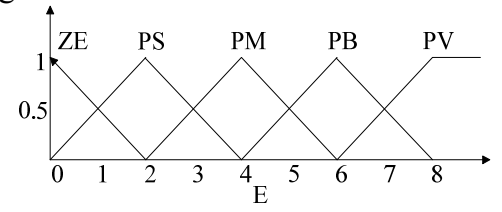

Figure 3. Membership function

In order to acquire fuzzy decision rules, the fuzzy rough set is used to attribute reductions. The extraction process can be finished as follows:

1) Reducing the condition attribution so as to delete redundant rules from decision table;

2) Deletes the repetitive rules;

3) Cancel redundant attribute values, and expresses the extracted rules in the form of IF ... THEN.

4) Determine the fuzzy rule base. The condition and conclusion of fuzzy production rules can be expressed as: IF (condition) Then (conclusion).

Thus the fuzzy rules of diagnostic and therapeutical tinnitus can be described as:
1) If the characteristic quantity of symptom is " $\mathrm{ZE}$ " and the characteristic quantity of eccentricity is " $\mathrm{ZE}$ ", then ear is normal;

2) If the characteristic quantity of symptom is "PS" and the characteristic quantity of eccentricity indication is "ordinary", then ear sound loudness grate is 1;

3 ) If the characteristic quantity of symptom is "PM" and the characteristic quantity of eccentricity indication is "a little", then ear sound loudness grate is 2 ;

4) If the characteristic quantity of symptom is "PB" and the characteristic quantity of eccentricity indication is "mild", then ear sound loudness grate is 3 ;

5 ) If the characteristic quantity of symptom is "PB" and the characteristic quantity of eccentricity indication is "moderate", then ear sound loudness grate is 4 ;

6) If the characteristic quantity of symptom is "PB" and the characteristic quantity of eccentricity indication is "severe", then ear sound loudness grate is 5;

7) If the characteristic quantity of symptom is "PB" and the characteristic quantity of eccentricity indication is "terrible", then ear sound loudness grate is 6;

8) If the characteristic quantity of symptom is "PB" and the characteristic quantity of eccentricity indication is "very terrible", then ear sound loudness grate is 7;

9) If the characteristic quantity of symptom is "PB" and the characteristic quantity of eccentricity indication is "deft", then ear sound loudness grate is 8 ;

In order to reduce the reluctant rules, the similarity degree threshold is selected as $\mathrm{K} \in[0,1]$. The each symptom is computed according to rough set theory to get the similarity class of tinnitus. According to the similarity degree computation, the classification results ban be obtained: $\{0\},\{1,2\},\{3,4,5\},\{6,7\},\{8\}$.

\section{SYSTEM REALIZATION}

An intelligence instrument for curing tinnitus is designed based on the former research results. The patients should consult doctor first. The doctor can refer patients for further tests if it is necessary. The process of detection and treatment in essence send the sound which is characteristic frequency voices and specific sound intensity level to patients. These voices can inhabit compensatory activity of the outer hair cells and relieve the symptom tinnitus. The outer hair cell resides outside the diseases of the cochlea. The sounds of this instrument can be divided into two categories, periodic signal and aperiodic signal. Periodic signal is realized by software programming. Single chip computer (C8051F410) and DDS (Direct Digital Frequency Synthesis) is this technology of main part. The aperiodic signals stored in the large memory of AT45DB161D.

Compared with Labwindows/CVI, the greatest advantage for $\mathrm{C}++$ Builder is the direct application of Microsoft ActiveX Data Object (ADO) and SQL Server. It completes with Data Aware Component. Thus, it is more convenient to store and query patient data from database. In this project, the upper computer uses visual programming environment 
$\mathrm{C}++$ Builder. So this software can perfectly combine full visualization with true object-oriented and the high efficiency and performance of $\mathrm{C}++$. It simplifies the development process without reducing the efficiency of executable code.

The multiple interface form of upper computer mainly include main interface, testing interface, treat interface, print interface, patient query interface, case record interface etc. The upper computer program is firmly in accordance with patient's treatment process. All the patients' data are stored into the database. In our project, we get curve from the Teechart control to analyze patients' testing data. Besides, the QRrport will work together with the database to generate our own statement easily. We apply VFW(Video for Windows) to edit patients photos to obtain Video Capture. AVICap window is used to support real-time video streaming and single frame capture and to control video source. Besides, in auricular treatment the animation is used to observe different voice wave produced by various prescription. The treatment interfaces in upper computer are shown in Fig. 4.

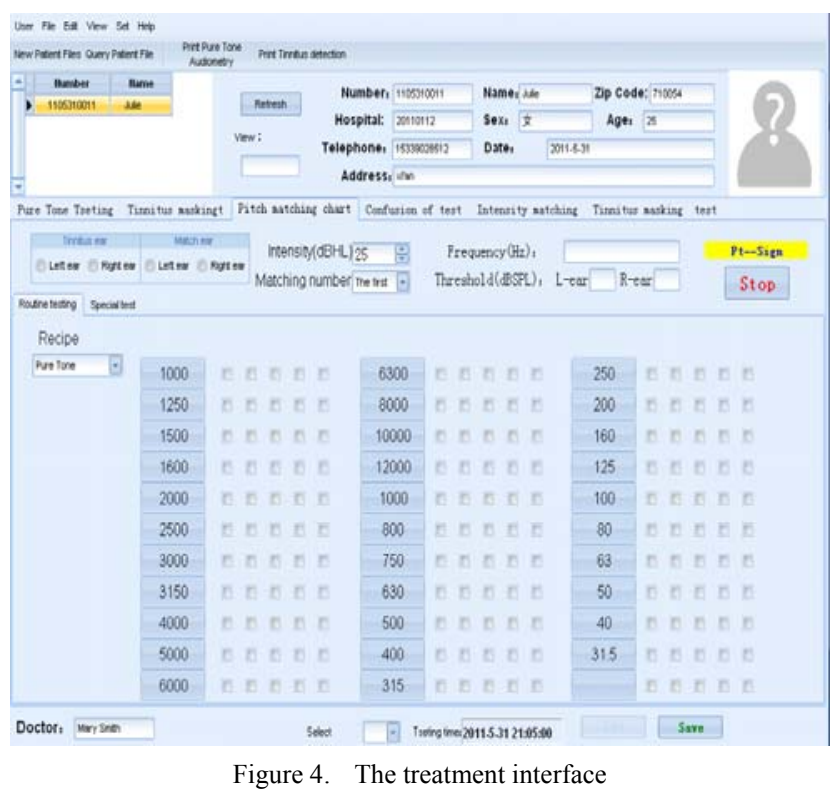

The intelligent Instrument has been put into operation in a hospital. Various technical indicators have reached the medical requirements. The whole system moves stably and works efficiently, the process is reasonable, and the function is completed. The masking board is shown in Fig. 5.

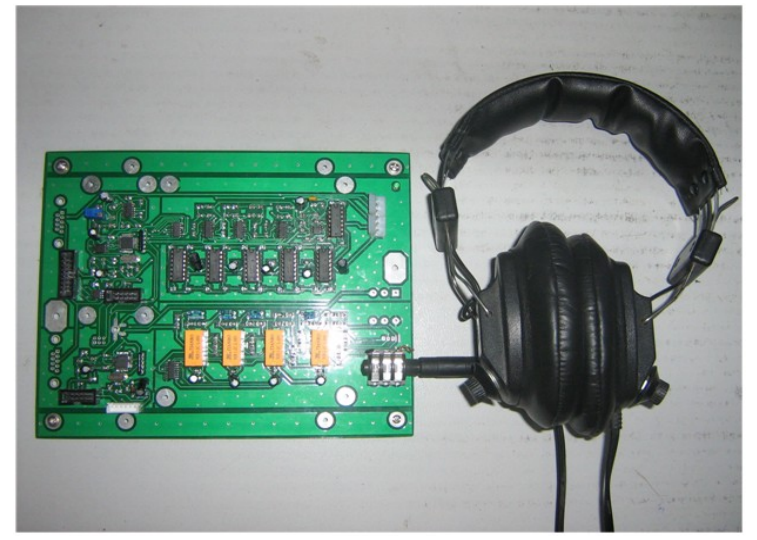

Figure 5. The masking board .

\section{CONCLUSION}

In this paper, a novel distributed system for the diagnostic and therapeutical tinnitus is introduced based on the fuzzy rough set theory. The knowledge representation system for tinnitus is introduced, and the attribute reduction and basic classical definitions of rough set are extended for the diagnostic and therapeutical tinnitus. Because the combination of rough sets and fuzzy sets are used, the method has the advantages of the classification, knowledge reduction, rule extraction, reduction and knowledge discovery for the diagnostic and therapeutical tinnitus system. The experimental results show convincingly that the diagnostic and therapeutical tinnitus process method based on fuzzy rough set theory achieves the better performance than the traditional process algorithms, because it is easier to acquire knowledge and extract rules. Therefore the problem of matching different clinical tinnitus is effectively solved, because the treatment prescription is of variety and selectivity.

\section{REFERENCES}

[1] HUANG Yan, YAN Guang-le, "Quantitative evaluation system for clinical efficacy of traditional Chinese medicine treatment", J. University of Shanghai for Science and Technology, Vol. 33 No. 1 2011, pp. 53-59.

[2] KANG Sheng-wul,WANG Ying-ming, CAI Zh-i feng1, "An Approach to Generating Rules Based on Rough and Fuzzy Sets Theories", Journal of Xiamen University (Natural Science), Vol. 41 No. 2, Mar. 2002, pp. 173-176.

[3] LIU Wen-jun, XIAO Qi-mei, "Fuzzy Decision Algorithm Based on Rough Sets", Fuzzy Systems and Mathematics, Vo 1. 20, No. 2, Apr . 2006, pp. 127-132.

[4] YU Da-ren, HU Qing-hua, BAO Wen, "Combining Rough Set Methodology And Fuzzy Clustering Forknowledge Discovery From Quantitative Data", Proceedings of the CSEE, Vol.24 No.6. Jun. 2004, pp.205-210. 